\title{
Local Control of Postinhibitory Rebound Spiking in CA1 Pyramidal Neuron Dendrites
}

\author{
Giorgio A. Ascoli, ${ }^{1,2 *}$ Sonia Gasparini, ${ }^{3 *}$ Virginia Medinilla, ${ }^{3}$ and Michele Migliore ${ }^{4}$ \\ ${ }^{1}$ Center for Neural Informatics, Structures, and Plasticity and ${ }^{2}$ Molecular Neuroscience Department, Krasnow Institute for Advanced Study, George Mason \\ University, Fairfax, Virginia 22030-4444, ${ }^{3}$ Neuroscience Center, Louisiana State University Health Sciences Center, New Orleans, Louisiana 70112, and \\ ${ }^{4}$ Institute of Biophysics, National Research Council, 90146 Palermo, Italy
}

Postinhibitory rebound spiking is characteristic of several neuron types and brain regions, where it sustains spontaneous activity and central pattern generation. However, rebound spikes are rarely observed in the principal cells of the hippocampus under physiological conditions. We report that CA1 pyramidal neurons support rebound spikes mediated by hyperpolarization-activated inward current $\left(I_{\mathrm{h}}\right)$, and normally masked by A-type potassium channels $\left(K_{\mathrm{A}}\right)$. In both experiments and computational models, $K_{\mathrm{A}}$ blockage or reduction consistently resulted in a somatic action potential upon release from hyperpolarizing injections in the soma or main apical dendrite. Rebound spiking was systematically abolished by the additional blockage or reduction of $I_{\mathrm{h}}$. Since the density of both $K_{\mathrm{A}}$ and $I_{\mathrm{h}}$ increases in these cells with the distance from the soma, such "latent" mechanism may be most effective in the distal dendrites, which are targeted by a variety of GABAergic interneurons. Detailed computer simulations, validated against the experimental data, demonstrate that rebound spiking can result from activation of distal inhibitory synapses. In particular, partial $K_{\mathrm{A}}$ reduction confined to one or few branches of the apical tuft may be sufficient to elicit a local spike following a train of synaptic inhibition. Moreover, the spatial extent and amount of $K_{\mathrm{A}}$ reduction determines whether the dendritic spike propagates to the soma. These data suggest that the plastic regulation of $K_{\mathrm{A}}$ can provide a dynamic switch to unmask postinhibitory spiking in CA1 pyramidal neurons. This newly discovered local modulation of postinhibitory spiking further increases the signal processing power of the CA1 synaptic microcircuitry.

\section{Introduction}

Postinhibitory rebound spiking has been characterized in several neuron types and brain regions, including cerebellar Purkinje cells (Llinás and Sugimori, 1980), thalamic relay cells (McCormick and Huguenard, 1992), neocortical spiny interneurons (Deuchars and Thomson, 1995), basal ganglia ventral pallidal neurons (Lavin and Grace, 1996), hypothalamic oxytocin-secreting cells (Armstrong and Stern, 1998), brainstem preBotzinger pacemakers (Del Negro et al., 2005), mesencephalic trigeminal neurons (Enomoto et al., 2006), and olfactory mitral cells (Balu and Strowbridge, 2007). Rebound spiking is believed to subserve crucial functions related to persistent activity and oscillatory dynamics, such as central pattern generation or sustained reverberations (Bottjer, 2005). Almost all studies that characterized the intrinsic membrane mechanisms underlying postinhibitory rebound reported a major role for voltagegated calcium conductances, often in conjunction with other ionic currents such as persistent sodium or the hyperpolarized-activated inward current $I_{\mathrm{h}}$.

Although rebound spiking was recently described in a subclass of "burst-non adapting narrow pyramidal neurons" in V1 (Le

\footnotetext{
Received Aug. 18, 2009; revised Feb. 10, 2010; accepted March 31, 2010.

This research was supported in part by National Institutes of Health R01 Grants AG25633 and NS39600 (to G.A.A.) and NSO35865 (to S.G.). We thank CINECA (Bologna, Italy) for granting access to their supercomputer system. *G.A.A. and S.G. contributed equally to this work.

Correspondence should be addressed to Giorgio A. Ascoli, Krasnow Institute for Advanced Study, MS2A1 George Mason University, 4400 University Drive, Fairfax, VA 22030-4444. E-mail: ascoli@gmu.edu.

D01:10.1523/JNEUROSCI.4066-09.2010

Copyright $\odot 2010$ the authors $\quad 0270-6474 / 10 / 306434-09 \$ 15.00 / 0$
}

Bon-Jego and Yuste, 2007), postinhibitory spikes are seldom observed in larger pyramidal cells. In the hippocampus, rebound spiking is more common in certain interneurons under normal physiological conditions (Lien et al., 2002), but has also been reported in principal cells after perisomatic GABAergic inhibition (Buhl et al., 1994). Moreover, CA1 pyramidal neurons may display rebound spiking in pathological models of febrile seizures (Chen et al., 2001) or in extreme conditions of very extended (10 s) hyperpolarizing somatic injections (Surges et al., 2006), as well as with specific parameter combinations in computational simulations of small circuits (Orbán et al., 2006). These studies suggest a prominent role of $I_{\mathrm{h}}$, which is expressed in high density in the distal dendrites of these neurons (Magee, 1998). Interestingly, the dendritic distribution of $I_{\mathrm{h}}$, increasing with the distance from the soma, is paralleled by A-type potassium channels $\left(K_{\mathrm{A}}\right)$, whose outward conductance is dynamically regulated at multiple time scales by highly plastic expression (Yuan and Chen, 2006), compartmentalization (Heusser and Schwappach, 2005, Kim et al., 2007) and phosphorylation by all major kinase pathways (Anderson et al., 2000; Varga et al., 2004; Rosenkranz et al., 2009; Schrader et al., 2009).

We hypothesized that $I_{\mathrm{h}}$ provides a mechanism for latent postinhibitory rebound spiking in CA1 pyramids that is usually silenced by $K_{\mathrm{A}}$, but can be unmasked by $K_{\mathrm{A}}$ reduction. In this work, we first used an existing computational model of CA1 pyramidal cells, without adjusting any biophysical parameter, to initially validate the hypothesis under standard assumptions. Next, we verified the hypothesis experimentally, extending the 


\section{a somatic injection}

sim. of hypothesis

(using 2005 model)

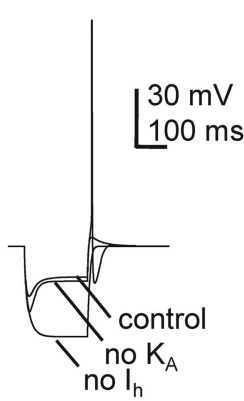

C

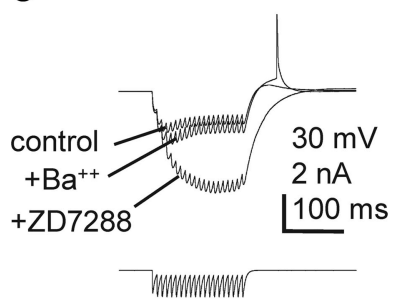

b

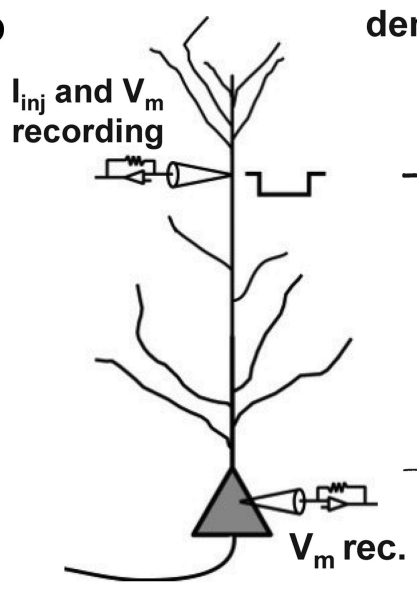

dendritic injection (exp)

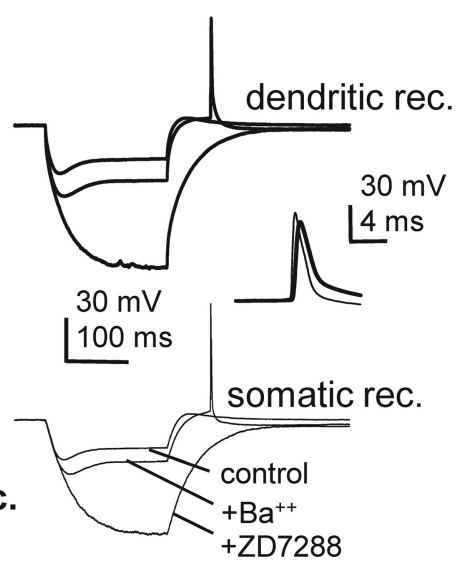

e d

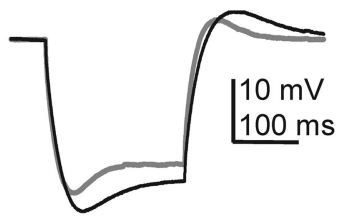

ctrl - rebound group

ctrl - no rebound

Figure 1. Postinhibitory rebound spiking in CA1 pyramidal neurons: hypothesis and experimental validation. $\boldsymbol{a}$, Simulated (left) and experimental (right) response to somatic hyperpolarization in control conditions, after blocking $K_{\mathrm{A}}$, and after blocking both $K_{\mathrm{A}}$ and $I_{\mathrm{h}}, \boldsymbol{b}$, Dual recordings of dendritic and somatic membrane potential obtained during a dendritic current injection ( $-1 \mathrm{nA}, 200$ $\mathrm{ms}$ at $\sim 250 \mu \mathrm{m}$ from the soma). The drawing on the left shows the experimental configuration. c, Voltage traces of the dendritic membrane potential recorded in response to a train (20 IPSCs at $100 \mathrm{~Hz})$ injected in the apical trunk at $300 \mu \mathrm{m}$ from the soma in control conditions, in the presence of $\mathrm{Ba}^{2+}(150 \mu \mathrm{M})$, and in the presence of $\mathrm{Ba}^{2+}$ and ZD $7288(20 \mu \mathrm{M})$. The current trace below represents the injected train of IPSCs. $\boldsymbol{d}$, Somatic voltage traces recorded in response to a $200 \mathrm{~ms},-400 \mathrm{pA}$ current injection to show the differences in sag and rebound from a neuron that shows rebound spiking (black trace) and one that does not (gray trace).e, Cumulative probability to have a rebound spike as a function of the time constant of the sag; symbols on the two sides of the curve show the average values ( \pm SEM) for the two groups of cells, those that show rebound firing (black) and those that do not (gray); the inset shows the number of neurons initiating a rebound spike as a function of the amplitude of somatic current injection.

initial conditions suggested by the simulation (somatic injection and recording) to dendritic injection/recording for testing the potential relevance of the mechanism to distal synapse activation. We therefore quantitatively tuned the model to faithfully reproduce the experimental data, creating stricter constraints for the following detailed simulations. In these conditions, we show that the proposed mechanism is viable under physiological GABAergic inhibition, and robust across wide variations of $I_{\mathrm{h}}$ expression and synaptic stimulation (input number and intensity). Furthermore, we demonstrate that local $K_{\mathrm{A}}$ reduction controls both initiation and propagation of postinhibitory dendritic spikes.

\section{Materials and Methods}

Electrophysiological experiments. Slices (400 $\mu \mathrm{m}$ thick) were prepared from 8- to 12-week old Sprague Dawley rats as previously described (Gasparini et al., 2007), according to methods approved by the Louisiana State University Health Sciences Center Institutional Animal Care and Use Committee. The external solution used for recordings was saturated with $95 \% \mathrm{O}_{2}$ and $5 \% \mathrm{CO}_{2}$ and contained the following (in $\mathrm{mm}$ ): $\mathrm{NaCl}$ $125, \mathrm{KCl} 2.5, \mathrm{NaHCO}_{3} 25, \mathrm{NaHPO}_{4} 1.25, \mathrm{CaCl}_{2} 2, \mathrm{MgCl}_{2} 1$, and D-glucose $25, \mathrm{pH}$ 7.4. CA1 pyramidal neurons were visualized using a Zeiss Axioskop equipped with infrared video microscopy and differential interference contrast (DIC) optics. Whole-cell patch-clamp recordings from the somata and dendrites (Fig. 1) were performed at $34-36^{\circ} \mathrm{C}$ using Dagan BVC-700 amplifiers in the active "bridge" mode. Patch pipettes had a resistance of 2-3 M $\Omega$ for somatic recordings and 4-6 M $\Omega$ for dendritic recordings when filled with a solution containing the following (in mM): K-methylsulfonate 130, HEPES 10, $\mathrm{NaCl} 4, \mathrm{Mg}_{2} \mathrm{ATP} 4$, $\mathrm{Tris}_{2}$ GTP 0.3 , phosphocreatine (di-Tris salt) 14, pH 7.3. The series resistance was generally $<20 \mathrm{M} \Omega$. The distance of the dendritic electrode from the visually identified soma was measured under DIC conditions. Hyperpolarizing steps or trains of IPSC-shaped currents characterized by $\mathrm{GABA}_{\mathrm{A}}$ current kinetics $\left(\tau_{\text {rise }} 0.5 \mathrm{~ms}, \tau_{\text {decay }} 5 \mathrm{~ms}\right.$ ) (Andrásfalvy and Mody, 2006) were injected through the whole-cell electrode. The current amplitude values reported in the text refer to the peak. In the experiments with 4-aminopyridine (4-AP), 6-cyano-7-nitroquinoxaline-2,3-dione (CNQX, $25 \mu \mathrm{M}$ ), DL-2-amino-5-phosphonopentanoic acid (DL-APV, 50 $\mu \mathrm{M})$ and gabazine $(12.5 \mu \mathrm{M})$ were added to the recording solutions to block AMPA and NMDA glutamatergic receptors and $\mathrm{GABA}_{\mathrm{A}}$ receptors, respectively, and to prevent the initiation of epileptiform activity (Perreault and Avoli, 1991).

Data are reported as means \pm SEM unless otherwise noted.

Computational modeling. All simulations were implemented with the NEURON program (v6.2) (Carnevale and Hines, 2006) using the variable time step feature. In some cases, a parallel version was run under MPI on an IBM Linux cluster (5120-processors, CINECA consortium). Model files are available for public download under the ModelDB section of the Senselab database (http://senselab.med.yale.edu). The first set of simulations used a preexisting CA1 pyramidal cell model (Migliore et al., 2005), downloaded from Senselab (accession number 55035), and used without alterations (Fig. 1a). In this previously described model, already validated against a number of different experimental findings on CA1 neurons (Migliore et al., 2005), sodium $\left(I_{\mathrm{Na}}\right)$ and delayed rectifier potas- 
sium $\left(K_{\mathrm{DR}}\right)$ conductances were uniformly distributed throughout the dendrites, while the $K_{\mathrm{A}}$ and $I_{\mathrm{h}}$ conductances linearly increased up to $500 \mu \mathrm{m}$ from the soma. The model contained a $100-\mu \mathrm{m}$-long axonal compartment that enabled realistic axosomatic spike generation. Its membrane biophysics was specified at the same level of realism as that of the dendrites.

In subsequent simulations, this initial model was modified to fit more specifically the experimental data of the present study (Fig. 1b). To this aim, two three-dimensional reconstructions of rat CA1 pyramidal neurons (Fig. 2a), cells pc2b (Megías et al., 2001) and ri06 (Golding et al., 2005), were selected from the public archive NeuroMorpho.Org (Ascoli et al., 2007). Except for $I_{\mathrm{h}}$ distribution, all voltagedependent ionic channels, kinetics, and membrane distributions for both neurons were left identical to those in the initial model. The passive properties and $I_{\mathrm{h}}$ distribution parameters were optimized to simultaneously fit both the somatic and dendritic responses to dendritic current injection under control conditions. In particular, $I_{\mathrm{h}}$ was inserted in all compartments and its peak conductance modeled as a sigmoidal increase with distance from the soma (Narayanan and Johnston, 2007) as follows:

$$
g_{\mathrm{h}}(x)=\bar{g}_{\mathrm{h}} \cdot\left[1+100 /\left(1+e^{\left(x_{0}-x\right) / s}\right)\right],
$$

where $\bar{g}_{\mathrm{h}}$ is the somatic density, $x$ is the distance from soma (in micrometers), and the constants $x_{0}$ and $s$ define the shape of the sigmoid (Fig. 2b). The values of the fitted parameters for both neurons, obtained using the Multiple Run Fitter tool of NEURON, are reported in Table 1. Since the geometries did not include dendritic spines, $R_{\mathrm{m}}$ was divided and $C_{\mathrm{m}}$ multiplied by a scaling value to account for spine density and the corresponding contribution to membrane surface area (Golding et al., 2005). In a subset of simulations (see Fig. $3 a), R_{\mathrm{a}}$ was increased by a factor of 2.8 to model the experimentally observed increase in the input resistance caused by $\mathrm{Ba}^{2+}$.

Because in this work we were specifically interested in investigating the interplay between $I_{\mathrm{h}}$ and $K_{\mathrm{A}}$ in modulating rebound spiking activity, we limited the types and numbers of different active membrane properties in the model. Several additional conductances reported in CA1 pyramidal cells, such as some voltage- and $\mathrm{Ca}^{2+}$-dependent $\mathrm{K}^{+}$, persistent $\mathrm{Na}^{+}$, and low-threshold $\mathrm{Ca}^{2+}$ currents, might further modulate the rebound process (Reyes, 2001). Their inclusion in the basic model, however, would generate unnecessary confusion in establishing a proof of principle of the hypothesized main mechanism underlying rebound spiking, especially in the distal dendrites. Nevertheless, in a selected set of simulations, low-threshold $\mathrm{Ca}^{2+}$ channels $\left(\mathrm{Ca}_{\mathrm{T}}\right)$ and a basic calcium extrusion mechanism were uniformly distributed throughout the neuron. In these cases, we used the model files from a previous paper (Shah et al., 2008). The resting $\mathrm{Ca}^{2+}$ concentration was set at $50 \mathrm{~nm}$, and the channel density was adjusted to obtain the $\sim 10 \mathrm{~nm}$ increase in somatic $\left[\mathrm{Ca}^{2+}\right]_{\mathrm{i}}$ after each action potential observed in pyramidal neurons (Lee et al., 2005). This choice assumes that approximately half of the $\mathrm{Ca}^{2+}$ entry occurs through these channels. In two simulations, performed to help with the interpretation of the experimental data obtained using different $K_{\mathrm{A}}$ blockers (see Fig. $3 b$ ), $K_{\mathrm{M}}$ was added in the soma (Shah et al., $2008)$ and $K_{\mathrm{D}}$ in the proximal apical trunk within $100 \mu \mathrm{m}$ from the soma [Metz et al. (2007); voltage dependence and kinetics from Hemond et al. (2008)], with peak densities of 5 and $1 \mu \mathrm{S} / \mathrm{cm}^{2}$, respectively. These two currents were not included in the model used for the main set of simulations (see Figs. 2, 4, 5). b

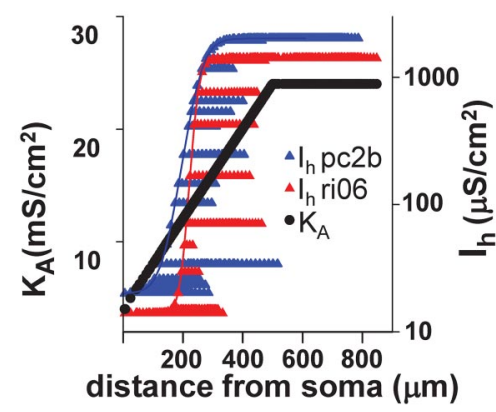

d

dendritic injection (model)

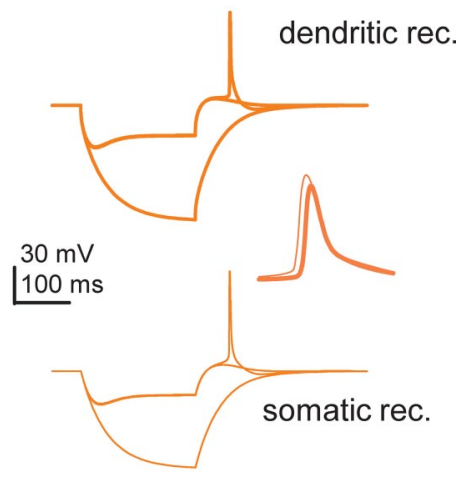

Table 1. Parameter values best fitting the experimental traces with the two model neurons

\begin{tabular}{llllllc}
\hline & $g_{\mathrm{h}}\left(\mu \mathrm{S} / \mathrm{cm}^{2}\right)$ & $x_{0}(\mu \mathrm{m})$ & $s(\mu \mathrm{m})$ & $R_{\mathrm{m}}\left(\mathrm{k} \Omega \cdot \mathrm{cm}^{2}\right)$ & $C_{\mathrm{m}}\left(\mu \mathrm{F} / \mathrm{cm}^{2}\right)$ & $R_{\mathrm{a}}(\Omega \cdot \mathrm{cm})$ \\
\hline pc2b & 20 & 249 & 12 & 14.6 & 3.2 & 50 \\
rio6 & 12 & 256 & 26 & 19 & 2.4 & 132 \\
\hline
\end{tabular}

Inhibitory synapses were uniformly distributed in all compartments beyond $400 \mu \mathrm{m}$ from the soma, resulting in 244 and 182 synapses for neurons $p c 2 b$ and ri06, respectively, consistent with experimental observations (Megías et al., 2001). Individual synaptic currents were modeled as a double-exponential conductance change with rise and decay times, respectively, of 0.5 and $5 \mathrm{~ms}$ (Andrásfalvy and Mody, 2006) and a reversal potential of $-75 \mathrm{mV}$ (Glickfeld et al., 2009). For every synapse, several values of peak conductances (1-4 nS) were tested, consistent with the range of reported measures (Hefft and Jonas, 2005; Glickfeld and Scanziani, 2006). During each simulation, the appropriate subset of synapses was activated at $200 \mathrm{~Hz}$ (Spampanato and Mody, 2007) in different parts of the distal dendritic tree with 10-30 stimuli for 50-150 ms to test the occurrence of a local and/or somatic rebound spike.

\section{Results}

Our basic hypothesis is that $I_{\mathrm{h}}$ provides a mechanism for latent postinhibitory rebound spiking in CA1 pyramidal neurons that is usually silenced by $K_{\mathrm{A}}$, but can be unmasked by $K_{\mathrm{A}}$ reduction. To quantitatively define and substantiate this hypothesis, we first used an existing computational model of CA1 pyramidal cells, previously validated against a number of standard conditions (Migliore et al., 2005), without altering any biophysical parame- 
ter. In particular, we hyperpolarized the soma with a square current injection in simulated current-clamp conditions, and recorded the resulting voltage. Although we always observed a postinhibitory rebound depolarization (Fig. 1a), under control conditions, no amount or duration of the hyperpolarization led to somatic spiking within a broad range of conditions (up to 500 $\mathrm{ms}$ and $-4 \mathrm{nA}$, corresponding to a peak somatic hyperpolarization of $-125 \mathrm{mV}$ ). In contrast, after removing $K_{\mathrm{A}}$ from the model, the hyperpolarizing somatic step was systematically followed by a rebound spike over the entire range of tested parameters for both duration and intensity of the current injection. However, further turning off $I_{\mathrm{h}}$ in the model completely abolished rebound spiking (Fig. $1 a$, left).

The hypothesis demonstrated in these initial simulations was then tested experimentally. We first performed a series of experiments to analyze the conditions for the generation of a rebound spike in CA1 pyramidal neurons. To this purpose, we injected variable hyperpolarizing current steps through somatic patchclamp electrodes (Fig. 1a, right). Neurons responded with a sag during the hyperpolarization and a depolarizing rebound at the end of the current injection, consistent with the slow activation and deactivation of $I_{\mathrm{h}}$ (Pape, 1996). A rebound spike could never be elicited in control conditions, regardless of the duration (100$500 \mathrm{~ms}$ ) and the amplitude (100-920 pA) of the hyperpolarizing current injected in the soma $(n=56)$. However, when $I_{\mathrm{A}}$ was partially blocked by bath perfusion of $\mathrm{Ba}^{2+}(150 \mu \mathrm{M})$ (Gasparini et al., 2007), a rebound spike could be elicited in most neurons ( 15 of 20 for a $200 \mathrm{~ms}, 300-600$ pA hyperpolarizing step, with an average of $-457 \pm 23 \mathrm{pA}, n=15)$.

Because the densities of $K_{\mathrm{A}}$ and $I_{\mathrm{h}}$ in the apical dendrites of CA1 pyramidal neurons increase with the distance from the soma (Hoffman et al., 1997; Magee, 1998), this mechanism could be particularly significant at more distal dendritic locations, with important consequences for gated transmission of the dendritic spike to the soma (Jarsky et al., 2005) and local plasticity phenomena (Golding et al., 2002; Magee and Johnston, 2005). Thus, we performed a series of experiments in which either a hyperpolarizing step or a train of 20 IPSC-shaped currents at $100 \mathrm{~Hz}$ was injected in the distal apical dendrite $(>240 \mu \mathrm{m}$ from the soma, for an average distance of $267 \pm 11 \mu \mathrm{m}, n=8)$ to simulate a barrage of synaptic inhibition (Fig. 1b,c). Under control conditions, none of the eight neurons tested showed a rebound spike for $200 \mathrm{~ms}$ hyperpolarizing steps up to $-2000 \mathrm{pA}$ and IPSCs up to $4200 \mathrm{pA}$. In the presence of $\mathrm{Ba}^{2+}(150 \mu \mathrm{M})$ to partially block $K_{\mathrm{A}}$ (Gasparini et al., 2007), seven of the eight neurons showed a rebound spike (Fig. 1b).

The hyperpolarizing current amplitude required to elicit a rebound spike in the presence of $\mathrm{Ba}^{2+}$ was on average $-1057 \pm$ $115 \mathrm{pA}$ in the case of the $200 \mathrm{~ms}$ current step $(n=7)$ and $-1990 \pm 230 \mathrm{pA}$ in the case of the IPSC train $(n=4)$ (Fig. $1 c$ ). The perfusion of ZD 7288 to block $I_{\mathrm{h}}$ abolished the rebound spikes in all neurons tested. In a subset of experiments, a second electrode was used to measure the somatic output of the CA1 neuron (Fig. 1b). These recordings showed that in these conditions, with the dendritic electrode injecting current in the main apical dendrite, the rebound spike was still initiated in the soma (always in the presence, but not in the absence, of $\mathrm{Ba}^{2+}$ ) and subsequently backpropagated in the apical dendrite, as visible from the superimposition of the somatic and dendritic voltage traces $(n=3)$ (Fig. $1 b$, inset). As shown before, the perfusion of ZD 7288 to block $I_{\mathrm{h}}$ prevented the rebound spike (Fig. $1 b, c$ ).

The neurons that displayed rebound spiking in the presence of $\mathrm{Ba}^{2+}(150 \mu \mathrm{M})$ during somatic hyperpolarizing current injec- tions were characterized by a sag with slower time constants than those that did not fire $(63.3 \pm 4.0 \mathrm{~ms}$ vs $34.8 \pm 4.9 \mathrm{~ms}$, measured for a $-400 \mathrm{pA}$ hyperpolarizing step of $200 \mathrm{~ms}, n=15$ and 5, respectively, $p<0.005$, Kolmogorov-Smirnov test) (Fig. 1d). This trend can be appreciated in Figure 1e, where the cumulative probability of firing is expressed as a function of time constant of the sag. This different behavior could be due to a different relative distribution of HCN1 and HCN2 subunits in the dendrites of the CA1 neurons examined (Brewster et al., 2007), since the two subunits are characterized by distinct activation kinetics (Santoro et al., 2000; Ulens and Tytgat, 2001). Interestingly, a gradient in the properties of $I_{\mathrm{h}}$ along the hippocampal axis has been recently suggested, with more functional HCN1 subunits in the dorsal hippocampus and more functional HCN2 subunits in the ventral hippocampus (Diaz and Johnston, 2009). Slower activating HCN2 subunits also display slower deactivation kinetics (van Welie et al., 2005); this feature could be reflected in a larger and/or longer rebound. We found that the area under the curve delimited by the rebound was larger for the group of neurons that showed rebound spiking $(0.32 \pm 0.01 \mathrm{mV} \cdot \mathrm{ms}$ vs $0.13 \pm 0.03$ $\mathrm{mV} \cdot \mathrm{ms}, n=15$ and 5, respectively, $p<0.001$, KolmogorovSmirnov test) (Fig. 1d). The smaller rebound depolarization generated by the neurons characterized by faster $I_{\mathrm{h}}$ kinetics could thus be responsible for the lack of rebound firing in a smaller population of neurons. Noticeably, the neurons that displayed rebound spikes in the presence of $\mathrm{Ba}^{2+}$ and those that did not failed to reveal statistically significant differences in the resting membrane potential $V_{\mathrm{m}}(-65.27 \pm 0.52 \mathrm{mV}$ vs $-65.74 \pm 1.38$ $\mathrm{mV})$, the peak hyperpolarization $(-24.87 \pm 0.84 \mathrm{mV}$ vs $-22.18 \pm 1.07 \mathrm{mV})$, the steady-state hyperpolarization $(-20.60 \pm 0.77 \mathrm{mV}$ vs $-18.85 \pm 0.58 \mathrm{mV})$, and the peak rebound potential measured from $V_{\mathrm{m}}(3.29 \pm 0.24 \mathrm{mV}$ vs $2.00 \pm$ $0.65 \mathrm{mV}$; in all four cases $p>0.1$, Kolmogorov-Smirnov test; parameters measured for a $200 \mathrm{~ms},-400 \mathrm{pA}$ hyperpolarizing current step). The fundamental role of $I_{\mathrm{h}}$ in initiating the spike was demonstrated by the fact that rebound spiking was completely abolished by the perfusion $(20 \mu \mathrm{M})$ of the $I_{\mathrm{h}}$ blocker ZD 7288 in all neurons tested. Together, these data confirm the hypothesis that $I_{\mathrm{h}}$ is responsible for the depolarizing phase that leads to rebound spikes in CA1 neurons, but that in control conditions this depolarization is dampened by the activation of $K_{\mathrm{A}}$.

We therefore tried to reproduce these experimental data using the model. The experimental curves in control conditions were fitted according to the morphology of two different neurons (Fig. $2 a$ ). The best-fitting model parameters (Table 1 ) and $I_{\mathrm{h}}$ distributions (Fig. $2 b$ ) were used in all subsequent simulations. Figure $2 c$ shows the superimposition of the experimental and model voltage traces after fitting.

The simulations reproduced the main experimental findings upon dendritic current injection (Fig. 2d). In particular, rebound spiking was (1) initiated only when $K_{\mathrm{A}}$ was partially blocked (i.e., reduced to $80 \%$ or less of control), (2) eliminated by the block of $I_{\mathrm{h}}$, and (3) initiated in the soma (Fig. 2d). In the model, however, we did not observe the increased hyperpolarization (with respect to control) that was experimentally recorded during $\mathrm{Ba}^{2+}$ application (compare experimental traces in Fig. $1 b$ and model traces in Fig. $2 d$ ). This was probably caused by an increase in input resistance due to the block by $\mathrm{Ba}^{2+}$ of additional $\mathrm{K}^{+}$conductances not included in the model, such as $K_{\mathrm{M}}$, inward rectifier $\mathrm{K}^{+}$ currents, and KCNK currents (Chatelain et al., 2005; Taverna et al., 2005; Gasparini et al., 2007). The block of $\mathrm{Ba}^{2+}$-sensitive $\mathrm{K}^{+}$ currents expressed in CA1 neurons and the consequent changes in input resistance could limit the proposed interpretation that 
the block of $K_{\mathrm{A}}$ is the main factor for the initiation of rebound spiking in our experimental conditions. However, the generation of a rebound spike in the model, even in the absence of the larger hyperpolarization observed in the experiments, demonstrates that, at least in principle, this phenomenon may be solely due to the interplay of $I_{\mathrm{h}}$ and $K_{\mathrm{A}}$, rather than resulting from an input resistance increase as caused by $\mathrm{Ba}^{2+}$ application. Furthermore, to explore the possibility that an increase in input resistance due to unspecific effects of $\mathrm{Ba}^{2+}$ could contribute to rebound spiking, we performed a series of simulations that reproduced changes in input resistance equivalent to those observed in the presence of $\mathrm{Ba}^{2+}$. Rebound spikes were never initiated under these conditions (Fig. 3a).

Starting from this model (cell $p c 2 b$ ), we also explored the effects of a faster time constant for $I_{\mathrm{h}}$. Under control conditions, a somatic current injection resulted in the typical $I_{\mathrm{h}}$-dependent sag, and produced a rebound spike after partial block of $K_{\mathrm{A}}$ (Fig. $3 a, 10 \%$ of $K_{\mathrm{A}}$ from control trace). When the sag time constant was reduced using a faster $I_{\mathrm{h}}$ time constant, a reduction in $K_{\mathrm{A}}$ did not produce rebound spiking (Fig. $3 a, I_{\mathrm{h}}$ time constant reduced by half, $10 \% K_{\mathrm{A}}$ trace). This finding suggests that rebound spiking is facilitated by slower $I_{\mathrm{h}}$ kinetics, confirming the experimental observation that cells showing a faster sag do not generate rebound spikes after $\mathrm{Ba}^{2+}$ application (Fig. 1d,e).

To further address the possibility that rebound spiking in the presence of $\mathrm{Ba}^{2+}$ could be due to the block of conductances other than $K_{\mathrm{A}}$, we performed a series of experiments with 4-aminopyridine (4-AP, $2 \mathrm{mM}$ ). While $\mathrm{Ba}^{2+}$ and 4-AP block various $\mathrm{K}^{+}$channels, the spectra of the channels blocked do not overlap except for A-type $\mathrm{K}^{+}$channels (Storm, 1990). The approach of using two different nonspecific blockers for $K_{\mathrm{A}}$ such as 4-AP and $\mathrm{Ba}^{2+}$ was made necessary by the fact that selective blockers such as heteropodatoxin-2 $(\mathrm{HpTx}-2)$ failed to block $K_{\mathrm{A}}$ in our experimental conditions. In particular, we found that HpTx-2 did not affect the $\mathrm{Ca}^{2+}$ transients associated with backpropagating spikes at distal dendritic locations in CA1 pyramidal neurons (data not shown), where the decrease in amplitude along the dendrites is mediated by an increase in the density of $K_{\mathrm{A}}$ (Hoffman et al., 1997). We attribute this finding to the possible incomplete penetration of $\mathrm{HpTx}-2$ in brain slices, as corroborated by the similar results of other researchers in CA1 pyramidal and ventral tegmental area neurons (personal communications from Drs. Dax Hoffman, Jeffrey Magee, Zayd Khaliq, and Bruce Bean).

Confirming our previous findings (Fig. 1), 200 ms hyperpolarizing steps in control conditions generated a sag during hyperpolarization and a depolarizing rebound (Fig. $3 b$ ), but a rebound firing was never initiated. The perfusion of 4-AP (2 mM) generated rebound spiking in 12 of the 14 neurons tested (for a $-454 \pm$ $16 \mathrm{pA}$ average hyperpolarizing step, $n=12$ ). The main role of $I_{\mathrm{h}}$ in the initiation of rebound spiking was again confirmed by the

\section{C subthreshold $\mathrm{K}_{\mathrm{A}}$ activation}

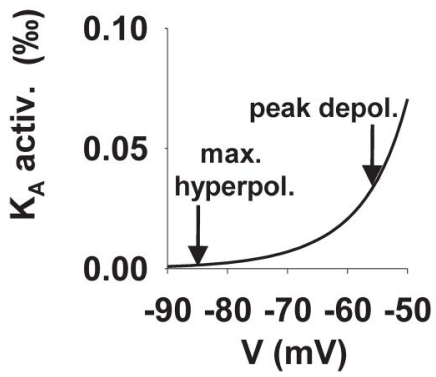

-AP, model with $\mathrm{K}_{\mathrm{M}}$ ctrl, higher $\mathrm{R}_{\mathrm{N}}$
ctrl, $10 \% \mathrm{~K}_{\mathrm{A}}$ $\tau_{h} / 2 I_{h}, 10 \% K_{A}$
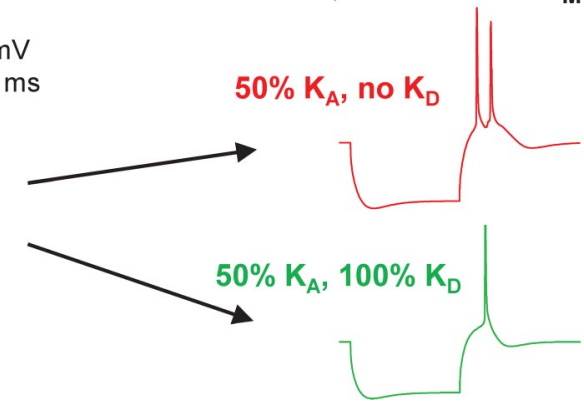

Figure 3. Dissecting the mechanism underlying rebound spiking in CA1 pyramidal cells. $\boldsymbol{a}$, Dendritic membrane potential from 7288 (blue); right, somatic voltage traces from simulations with the model including $K_{M}$, with (bottom, green) or without (top, red) $K_{D}$. c, Plot of normalized $K_{\mathrm{A}}$ activation in the subthreshold range of membrane potential.

fact that the spikes were abolished by perfusion of ZD 7288 (Fig. $3 b$ ). The input resistance was unaffected in 6 of 12 neurons in the presence of 4-AP, while a small increase in the steady-state hyperpolarization was observed in the other 6 neurons (ranging from 1 to $4.5 \mathrm{mV}$ for a -400 to $-500 \mathrm{pA}$ hyperpolarizing step). These data, together with the simulations in Figure $3 a$, argue against an influence in changes of the input resistance on the initiation of rebound spiking. However, the rebound spiking generated by 4 -AP was more complex than that initiated by $\mathrm{Ba}^{2+}$, since most neurons (9 of 12) showed a complex pattern of two or more spikes (Fig. $3 b$ ). We attributed this finding to the fact that 4-AP blocked the dendrotoxin-sensitive $K_{\mathrm{D}}$ in addition to $K_{\mathrm{A}}$ (Storm, 1988 ). Blockage of $K_{\mathrm{D}}$ could unmask the effect of the $K_{\mathrm{M}}$ conductance, thus causing complex firing (Metz et al., 2007). To explore this possibility, we conducted a series of simulations to show the differential effect of blocking $K_{\mathrm{A}}$ and $K_{\mathrm{D}}$ (Fig. $3 b$ ) on rebound spiking. For this particular set of simulation, $K_{\mathrm{D}}$ and $K_{\mathrm{M}}$ were added to the model neuron, as explained in Materials and Methods. Blockage of all of $K_{\mathrm{D}}$ and half of $K_{\mathrm{A}}$, simulating the effect of 2 mM 4-AP, elicited complex rebound spiking upon release from hyperpolarization (Fig. $3 b$, top right), very similar to that observed in our experimental conditions (Fig. $3 b$, left). However, selectively reducing $K_{\mathrm{A}}$ by $50 \%$, leaving $K_{\mathrm{D}}$ unaffected, resulted in the initiation of a single rebound spike (Fig. $3 b$, bottom right), similarly to our initial model and to the experimental data with $\mathrm{Ba}^{2+}$. Together, the experimental data and simulations show that a decrease in $K_{\mathrm{A}}$ plays a major role in the initiation of rebound spiking in CA1 pyramidal neurons.

Both model and experiments indicate that $K_{\mathrm{A}}$ activation at subthreshold potentials normally balances activation of $I_{\mathrm{h}}$, pre- 
venting rebound firing. Thus, if $K_{\mathrm{A}}$ is active subthreshold and blocked by 4-AP, it might appear surprising, in light of the clear effect of this blocker on the rebound firing, that 4-AP does not affect the input resistance in simulations and experiments alike. The reason can be found in the steep voltage dependence of $K_{\mathrm{A}}$ activation around the resting potential (Fig. 3c). At the peak rebound depolarization (approximately $-54 \mathrm{mV}$ ), enough channels are open to compensate for $I_{\mathrm{h}}$ current, unless these channels are blocked. In contrast, at or below resting potential, and in particular at the hyperpolarized voltages reported above (approximately $-85 \mathrm{mV}$ ), essentially all $K_{\mathrm{A}}$ channels are closed, therefore their blockage would not affect the input resistance at this level.

Next, we used this model (as described in Fig. 2) to explore the possibility of synaptically based activation of postinhibitory rebound spiking (Fig. 4). In particular, we examined the response to GABAergic signals on the distal apical tuft, the target of several CA1 interneurons (Klausberger and Somogyi, 2008), including oriens/lacunosum-moleculare, perforant-path-associated, neurogliaform, and radiatum-retrohippocampal projection cells. Simulations indicated that even a partial reduction of $K_{\mathrm{A}}$ (e.g., with $40 \%$ of the control conductance maintained) confined to the region of synaptic activation (e.g., single branches) may be sufficient to enable a dendritic rebound spike following release from inhibition. This local spike may or may not propagate to the soma depending on the dendrite morphology and the amount of $K_{\mathrm{A}}$ reduction (Fig. $4 a$ ).

Some modulation of these effects could be caused by fast, transient, low-threshold calcium channels $\left(\mathrm{Ca}_{\mathrm{T}}\right)$. Thus, we performed additional simulations with $\mathrm{Ca}_{\mathrm{T}}$ channels included in the model. From a series of 100 simulations, randomly redistributing 25 synapses over the entire tuft and using a $K_{\mathrm{A}}$ reduced to $40 \%$ of control, we calculated the minimum average peak inhibitory conductance resulting in a rebound spike in the tuft with or without $\mathrm{Ca}_{\mathrm{T}}$. The average values were very close (control: $2.0 \pm 0.08 \mathrm{nS}$; with $\mathrm{Ca}_{\mathrm{T}}: 1.94 \pm 0.07 \mathrm{nS}$ ) and their difference statistically not significant (Mann-Whitney rank sum test, $p>0.5$ ). One additional mechanism that can modulate the rebound process is the reversal potential of $\mathrm{GABA}_{\mathrm{A}}$ currents, that can vary from values close to the cell resting potential (Gulledge and Stuart, 2003) to approximately $-80 \mathrm{mV}$ (Hevers and Lüddens, 1998). With the same protocol used to test the effect of $\mathrm{Ca}_{\mathrm{T}}$ channels, we computed the minimum average reversal potential at which no rebound spike could be obtained. With the $K_{\mathrm{A}}$ reduced in the tuft to $15 \%$ of control, and $4 \mathrm{nS}$ for the individual peak synaptic conductance, the simulations indicated a threshold value of $-71.00 \pm 1.12 \mathrm{mV}\left(-71.06 \pm 1.07 \mathrm{mV}\right.$, with $\left.\mathrm{Ca}_{\mathrm{T}}\right)$, meaning that a rebound will be obtained for any $E_{\mathrm{Cl}}$ more hyperpolarized than this value.

Different inhibitory cells evoke synaptic patterns with diverse characteristics of firing frequency and durations. Thus we tested the conditions to initiate a dendritic rebound spike after partial reduction of the local $K_{\mathrm{A}}$ with different combinations of synaptic train duration and peak synaptic conductance. Moreover, to assess the sensitivity of the results to $I_{\mathrm{h}}$ density, we also varied this parameter from the initial best-fitting value. As expected, shorter train duration, i.e., fewer presynaptic action potentials, required larger synaptic conductance (Fig. $4 b$ ), possibly corresponding to the synchronous coactivation of a larger number of inputs. Nevertheless, rebound spiking was robust over a twofold change of $I_{\mathrm{h}}$ density (somatic values: $20-40 \mu \mathrm{S} / \mathrm{cm}^{2}$ ). Similarly, we explored the effect of a broader range of the time constant of the IPSCs $(5-20 \mathrm{~ms})$ and of the inhibitory firing frequency $(50-200 \mathrm{~Hz})$,
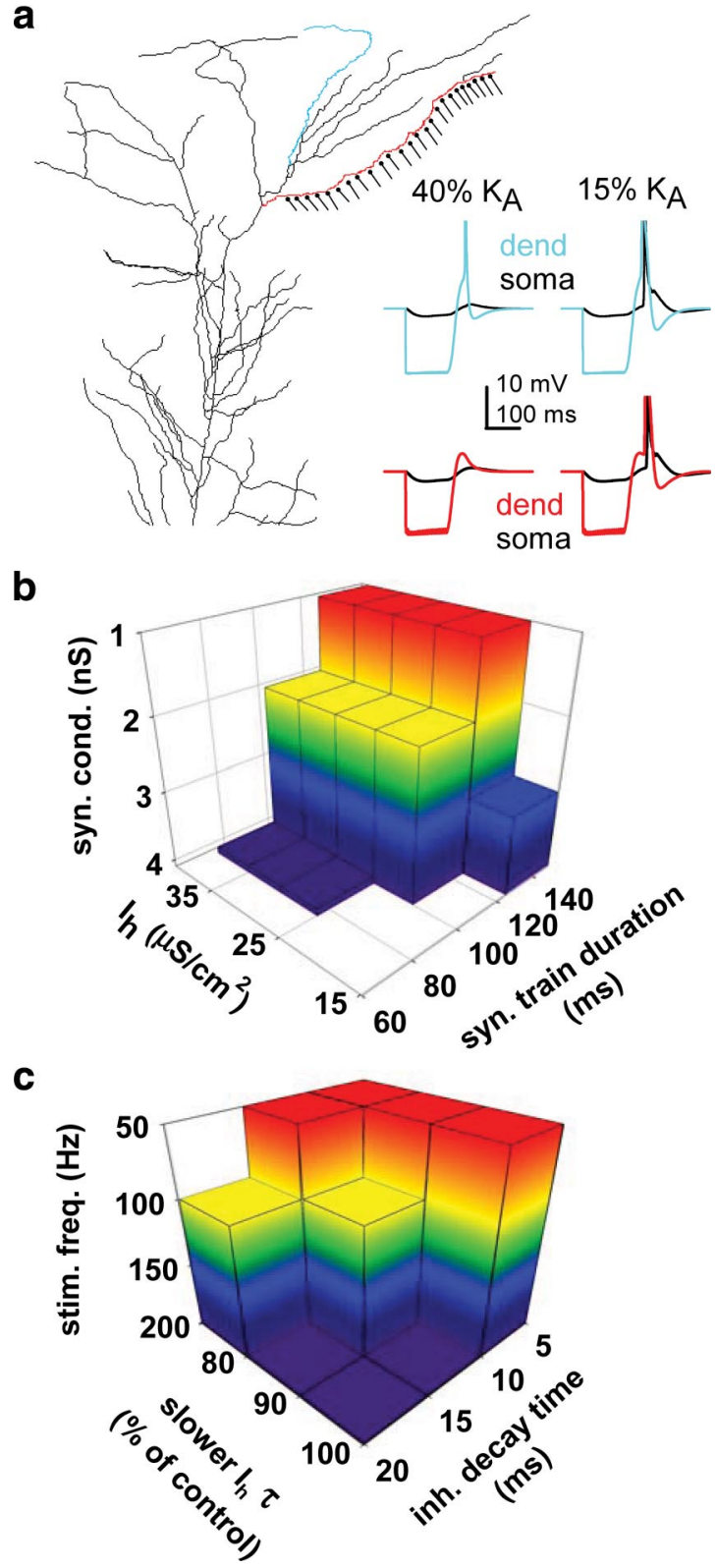

Figure 4. Local modulation of $K_{\mathrm{A}}$ can result in a dendritic rebound spike that may or not propagate to the soma. $\boldsymbol{a}$, Typical results obtained stimulating two different stretches of dendritic membrane (blue, $280 \mu \mathrm{m}^{2}$; red, $368 \mu \mathrm{m}^{2}$ ) in the same neuron $(p c 2 b)$, with the same number of synapses ( $n=25$, schematically indicated on the red branch). In both cases, somatic and dendritic membrane potentials (traces on the right) are plotted after different reduction ( $15 \%$ or $40 \%$ of control) of local $K_{A}$ channels. $\boldsymbol{b}$, Conditions to initiate a dendritic rebound spike in the blue branch with different combinations of the peak synaptic conductance, $I_{\mathrm{h}}$ somatic density, and synaptic train duration after reducing the local $K_{\mathrm{A}}$ to $40 \%$ of control. Equal colors represent combinations with the same peak synaptic conductance. $c$, Conditions required to initiate a dendritic rebound spike in the blue or in the red branch with different combinations of the stimulation frequency, $I_{\mathrm{h}}$ time constant, and decay time constant of the inhibitory synapses. Equal colors represent combinations with the same stimulation frequency; in both branches, synapses were activated 20 times, with the local $K_{A}$ reduced to $15 \%$ of control.

varying at the same time the $I_{\mathrm{h}}$ kinetics within the experimental observations. These simulations (Fig. $4 c$ ) determined that rebound spiking is a robust phenomenon.

Finally, we characterized the distinct conditions for local and propagating spikes in terms of spatial extent and amount of $K_{\mathrm{A}}$ inactivation (Fig. 5). In particular, we activated inhibitory synapses on all combinations of three or five adjacent branches in the 


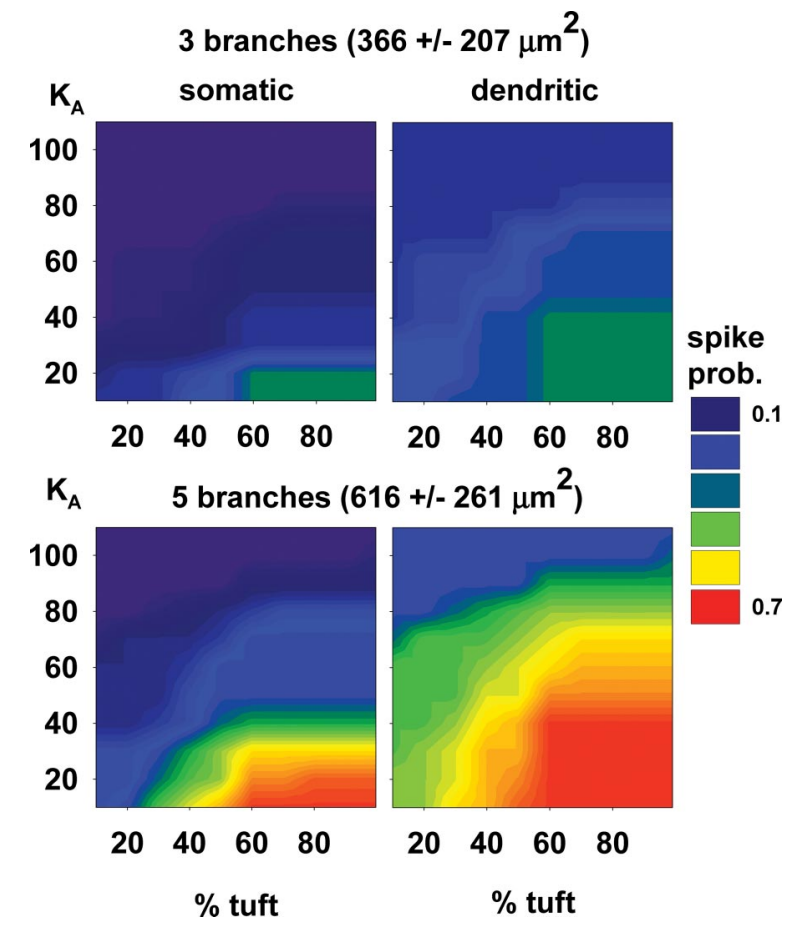

Figure 5. Average dendritic or somatic spike probability as a function of local $K_{\mathrm{A}}$ channel density (\% of control) and amount of the tuft on which $K_{A}$ is reduced. Synaptic stimulation was localized in contiguous branches of different sizes. The average spike probability was calculated by testing all combinations of three (top panels) and five (bottom panels) branches in neuron $p c 2 b$.

two morphological reconstructions. In each combination, we varied the amount of $K_{\mathrm{A}}$ reduction (in terms of residual peak conductance relative to control) and the proportion of the tuft in which $K_{\mathrm{A}}$ was reduced, starting from the synaptically activated branch and progressively involving greater surrounding dendritic areas. For each given pair of values for the spatial extent and amount of $K_{\mathrm{A}}$ inactivation, we expressed the fractions of all threebranch and five-branch combinations in which postinhibitory rebound led to a local dendritic spike that did not propagate or to a spike that propagated to the soma as corresponding "spike probabilities." Both the spatial extent and amount of $K_{\mathrm{A}}$ reduction independently increased the local spike probability, as did the amount of dendritic inhibition (from three to five branches). Among these factors, the amount of $K_{\mathrm{A}}$ reduction was most influential. More extreme conditions also enabled the local spike to propagate to the soma. However, even relatively "mild" conditions (e.g., $50 \%$ of inactivation in $50 \%$ of the tuft) yielded local spikes in a large proportion of the branch combinations. Note that these simulation results relate specifically to synaptic activity in the terminal tuft of the apical tree, whose dendritic branches are located in stratum lacunosum-moleculare (at $>400 \mu \mathrm{m}$ from the soma). In these conditions, the rebound spike initiation always occurs in the vicinity of the inhibitory synaptic input, and may or may not propagate to the soma. This phenomenon is distinct from that observed upon rebound from hyperpolarization of the main apical trunk located in stratum radiatum (between 240 and $350 \mu \mathrm{m}$ from the soma). In that case, the action potential is first generated in the soma rather than in the dendrites both in the experiments (Fig. 1b) and simulations (Fig. 2d).

\section{Discussion}

Neuronal computation is sculpted by the dendritic interplay between synaptic microcircuitry and active channel distribution. In
CA1 pyramidal neurons, several voltage-dependent conductances, including $K_{\mathrm{A}}$ and $I_{\mathrm{h}}$, display nonuniform densities that increase with the dendritic distance from the soma (Spruston, 2008). The dendrites of these cells are contacted by a diversity of GABAergic interneurons whose activity is phase locked to specific rhythms (Klausberger and Somogyi, 2008). Interneurons specifically targeting the distal tuft (where the expression of $K_{\mathrm{A}}$ and $I_{\mathrm{h}}$ is highest) include oriens/lacunosum-moleculare, perforant-pathway-associated, and neurogliaform cells (Maccaferri and Lacaille, 2003; Tamás et al., 2003). The exact number, receptor properties, and spontaneous in vivo activity of these inhibitory synapses on CA1 pyramidal neurons have not yet been conclusively determined for each of these classes. The plausible range of conditions we simulated, consistent with available data, suggest that rebound spiking could be elicited synaptically under physiological conditions.

Rebound spike in most neurons is due to the activity of transient calcium and sodium currents (McCormick and Huguenard, 1992; Zhang et al., 2004). Although $K_{\mathrm{A}}$ was occasionally reported to dampen spiking after strong inhibition (Balu and Strowbridge, 2007), it would not entirely abolish rebound spikes. We showed here that in CA1 pyramidal neurons, rebound spiking only requires $I_{\mathrm{h}}$, and is fully masked by $K_{\mathrm{A}}$. When $K_{\mathrm{A}}$ is blocked in the entire neuron, the initiation site for the rebound spike depends on the location of the inhibitory input. Hyperpolarization of the main apical dendrite (stratum radiatum) results in a somatic spike that backpropagates into the dendrites; inhibition of the tuft (stratum lacunosum-moleculare) elicits a local dendritic spike, which may or may not propagate to the soma. This distinction suggests that different interneuron subtypes might play separate functional roles in controlling the initiation and propagation of rebound spikes. In particular, Ivy, Schaffer collateral-associated, bistratified, trilaminar, and apical dendritic innervating cells mainly establish their synaptic contacts in stratum radiatum, while oriens/lacunosum-moleculare, perforant-pathway-associated, radiatum-retrohippocampal projection, and neurogliaform cells primarily target the distal tuft (Klausberger and Somogyi, 2008).

Mechanistically, the observations described in this report can be explained by the similar distributions of $I_{\mathrm{h}}$ and $K_{\mathrm{A}}$, whose densities increase from the soma to the distal dendrites. Functionally, modulating rebound spikes by $K_{\mathrm{A}}$ may be important because of the extensive local regulation of these potassium channels (Frick et al., 2003, 2004) by second messenger and phosphorylation cascades. In particular, the activity of $K_{\mathrm{A}}$ has been shown to be downregulated by neurotransmitters that activate PKA (protein kinase A) and PKC (protein kinase C), such as the noradrenergic agonist isoproterenol and the muscarinic agonist carbachol, respectively (Hoffman and Johnston, 1998, 1999). These modulators appear to converge on the MAPK (mitogen-activated protein kinase) pathway (Yuan et al., 2002) to regulate the activity of $K_{\mathrm{A}}$. Furthermore, plasticity phenomena such as long-term potentiation have been shown to increase dendritic excitability and spike backpropagation by downregulating $K_{\mathrm{A}}$ (Frick et al., 2004; Losonczy et al., 2008). This downregulation is achieved both by a leftward shift in the inactivation curve of $K_{\mathrm{A}}$ (Frick et al., 2004) and by the internalization of the channels that underlie $K_{\mathrm{A}}$ (Kim et al., 2007).

We showed that the amount and spatial extent of $K_{\mathrm{A}}$ reduction could gate not only the initiation of local dendritic spikes, but also their propagation to the soma. From this viewpoint, rebound spiking elicited by distal synaptic inhibition can provide a versatile signal for persistent changes in both local and global 
excitability (Zhang et al., 2004). In turn, such a "latent" mechanism can be relevant to the overall plasticity of these neurons and their ability to form cell assemblies (Chik et al., 2004), which provide a crucial substrate for memory encoding in the hippocampus (Harris, 2005).

\section{References}

Anderson AE, Adams JP, Qian Y, Cook RG, Pfaffinger PJ, Sweatt JD (2000) Kv4.2 phosphorylation by cyclic AMP-dependent protein kinase. J Biol Chem 275:5337-5346.

Andrásfalvy BK, Mody I (2006) Differences between the scaling of miniature IPSCs and EPSCs recorded in the dendrites of CA1 mouse pyramidal neurons. J Physiol 576:191-196.

Armstrong WE, Stern JE (1998) Phenotypic and state-dependent expression of the electrical and morphological properties of oxytocin and vasopressin neurones. Prog Brain Res 119:101-113.

Ascoli GA, Donohue DE, Halavi M (2007) NeuroMorpho.Org: a central resource for neuronal morphologies. J Neurosci 27:9247-9251.

Balu R, Strowbridge BW (2007) Opposing inward and outward conductances regulate rebound discharges in olfactory mitral cells. J Neurophysiol 97:1959-1968.

Bottjer SW (2005) Timing and prediction the code from basal ganglia to thalamus. Neuron 46:4-7.

Brewster AL, Chen Y, Bender RA, Yeh A, Shigemoto R, Baram TZ (2007) Quantitative analysis and subcellular distribution of mRNA and protein expression of the hyperpolarization-activated cyclic nucleotide-gated channels throughout development in rat hippocampus. Cereb Cortex 17:702-712.

Buhl EH, Halasy K, Somogyi P (1994) Diverse sources of hippocampal unitary inhibitory postsynaptic potentials and the number of synaptic release sites. Nature 368:823-828.

Carnevale NT, Hines ML (2006) The NEURON book. Cambridge, UK: Cambridge UP.

Chatelain FC, Alagem N, Xu Q, Pancaroglu R, Reuveny E, Minor DL Jr (2005) The pore helix dipole has a minor role in inward rectifier channel function. Neuron 47:833-843.

Chen K, Aradi I, Thon N, Eghbal-Ahmadi M, Baram TZ, Soltesz I (2001) Persistently modified h-channels after complex febrile seizures convert the seizure-induced enhancement of inhibition to hyperexcitability. Nat Med 7:331-337.

Chik DT, Coombes S, Wang ZD (2004) Clustering through postinhibitory rebound in synaptically coupled neurons. Phys Rev E Stat Nonlin Soft Matter Phys 70:011908.

Del Negro CA, Morgado-Valle C, Hayes JA, Mackay DD, Pace RW, Crowder EA, Feldman JL (2005) Sodium and calcium current-mediated pacemaker neurons and respiratory rhythm generation. J Neurosci 25:446-453.

Deuchars J, Thomson AM (1995) Innervation of burst firing spiny interneurons by pyramidal cells in deep layers of rat somatomotor cortex: paired intracellular recordings with biocytin filling. Neuroscience 69:739-755.

Diaz LM, Johnston D (2009) Pharmacological dissociation of h-channels in CA1 neurons between dorsal and ventral hippocampus of adult rats. Soc Neurosci Abstr 35:716.14.

Enomoto A, Han JM, Hsiao CF, Wu N, Chandler SH (2006) Participation of sodium currents in burst generation and control of membrane excitability in mesencephalic trigeminal neurons. J Neurosci 26:3412-3422.

Frick A, Magee J, Koester HJ, Migliore M, Johnston D (2003) Normalization of $\mathrm{Ca}^{2+}$ signals by small oblique dendrites of CA1 pyramidal neurons. J Neurosci 23:3243-3250.

Frick A, Magee J, Johnston D (2004) LTP is accompanied by an enhanced local excitability of pyramidal neuron dendrites. Nat Neurosci 7:126-135.

Gasparini S, Losonczy A, Chen X, Johnston D, Magee JC (2007) Associative pairing enhances action potential back-propagation in radial oblique branches of CA1 pyramidal neurons. J Physiol 580:787-800.

Glickfeld LL, Scanziani M (2006) Distinct timing in the activity of cannabinoid-sensitive and cannabinoid-insensitive basket cells. Nat Neurosci 9:807-815.

Glickfeld LL, Roberts JD, Somogyi P, Scanziani M (2009) Interneurons hyperpolarize pyramidal cells along their entire somatodendritic axis. Nat Neurosci 12:21-23.

Golding NL, Staff NP, Spruston N (2002) Dendritic spikes as a mechanism for cooperative long-term potentiation. Nature 418:326-331.
Golding NL, Mickus TJ, Katz Y, Kath WL, Spruston N (2005) Factors mediating powerful voltage attenuation along CA1 pyramidal neuron dendrites. J Physiol 568:69-82.

Gulledge AT, Stuart GJ (2003) Excitatory actions of GABA in the cortex. Neuron 37:299-309.

Harris KD (2005) Neural signatures of cell assembly organization. Nat Rev Neurosci 6:399-407.

Hefft S, Jonas P (2005) Asynchronous GABA release generates long-lasting inhibition at a hippocampal interneuron-principal neuron synapse. Nat Neurosci 8:1319-1328.

Hemond P, Epstein D, Boley A, Migliore M, Ascoli GA, Jaffe DB (2008) Distinct classes of pyramidal cells exhibit mutually exclusive firing patterns in hippocampal area CA3b. Hippocampus 18:411-424.

Heusser K, Schwappach B (2005) Trafficking of potassium channels. Curr Opin Neurobiol 15:364-369.

Hevers W, Lüddens H (1998) The diversity of GABAA receptors. Pharmacological and electrophysiological properties of GABAA channel subtypes. Mol Neurobiol 18:35-86.

Hoffman DA, Johnston D (1998) Downregulation of transient $\mathrm{K}^{+}$channels in dendrites of hippocampal CA1 pyramidal neurons by activation of PKA and PKC. J Neurosci 18:3521-3528.

Hoffman DA, Johnston D (1999) Neuromodulation of dendritic action potentials. J Neurophysiol 81:408-411.

Hoffman DA, Magee JC, Colbert CM, Johnston D (1997) $\mathrm{K}^{+}$channel regulation of signal propagation in dendrites of hippocampal pyramidal neurons. Nature 387:869-875.

Jarsky T, Roxin A, Kath WL, Spruston N (2005) Conditional dendritic spike propagation following distal synaptic activation of hippocampal CA1 pyramidal neurons. Nat Neurosci 8:1667-1676.

Kim J, Jung SC, Clemens AM, Petralia RS, Hoffman DA (2007) Regulation of dendritic excitability by activity-dependent trafficking of the A-type $\mathrm{K}^{+}$channel subunit Kv4.2 in hippocampal neurons. Neuron 54:933-947.

Klausberger T, Somogyi P (2008) Neuronal diversity and temporal dynamics: the unity of hippocampal circuit operations. Science 321:53-57.

Lavin A, Grace AA (1996) Physiological properties of rat ventral pallidal neurons recorded intracellularly in vivo. J Neurophysiol 75:1432-1443.

Le Bon-Jego M, Yuste R (2007) Persistently active, pacemaker-like neurons in neocortex. Front Neurosci 1:123-129.

Lee JC, Callaway JC, Foehring RC (2005) Effects of temperature on calcium transients and $\mathrm{Ca}^{2+}$-dependent afterhyperpolarizations in neocortical pyramidal neurons. J Neurophysiol 93:2012-2020.

Lien CC, Martina M, Schultz JH, Ehmke H, Jonas P (2002) Gating, modulation and subunit composition of voltage-gated $\mathrm{K}(+)$ channels in dendritic inhibitory interneurones of rat hippocampus. J Physiol 538:405-419.

Llinás R, Sugimori M (1980) Electrophysiological properties of in vitro Purkinje cell somata in mammalian cerebellar slices. J Physiol 305:171-195.

Losonczy A, Makara JK, Magee JC (2008) Compartmentalized dendritic plasticity and input feature storage in neurons. Nature 452:436-441.

Maccaferri G, Lacaille JC (2003) Interneuron diversity series: hippocampal interneuron classifications - making things as simple as possible, not simpler. Trends Neurosci 26:564-571.

Magee JC (1998) Dendritic hyperpolarization-activated currents modify the integrative properties of hippocampal CAl pyramidal neurons. J Neurosci 18:7613-7624.

Magee JC, Johnston D (2005) Plasticity of dendritic function. Curr Opin Neurobiol 15:334-342.

McCormick DA, Huguenard JR (1992) A model of the electrophysiological properties of thalamocortical relay neurons. J Neurophysiol 68:1384-1400

Megías M, Emri Z, Freund TF, Gulyás AI (2001) Total number and distribution of inhibitory and excitatory synapses on hippocampal CA1 pyramidal cells. Neuroscience 102:527-540.

Metz AE, Spruston N, Martina M (2007) Dendritic D-type potassium currents inhibit the spike afterdepolarization in rat hippocampal CA1 pyramidal neurons. J Physiol 581:175-187.

Migliore M, Ferrante M, Ascoli GA (2005) Signal propagation in oblique dendrites of CA1 pyramidal cells. J Neurophysiol 94:4145-4155.

Narayanan R, Johnston D (2007) Long-term potentiation in rat hippocampal neurons is accompanied by spatially widespread changes in intrinsic oscillatory dynamics and excitability. Neuron 56:1061-1075.

Orbán G, Kiss T, Érdi P (2006) Intrinsic and synaptic mechanisms deter- 
mining the timing of neuron population activity during hippocampal theta oscillation. J Neurophysiol 96:2889-2904.

Pape HC (1996) Queer current and pacemaker: the hyperpolarizationactivated cation current in neurons. Annu Rev Physiol 58:299-327.

Perreault P, Avoli M (1991) Physiology and pharmacology of epileptiform activity induced by 4 -aminopyridine in rat hippocampal slices. J Neurophysiol 65:771-785.

Reyes A (2001) Influence of dendritic conductances on the input-output properties of neurons. Annu Rev Neurosci 24:653-675.

Rosenkranz JA, Frick A, Johnston D (2009) Kinase-dependent modification of dendritic excitability after long-term potentiation. J Physiol 587:115-125.

Santoro B, Chen S, Luthi A, Pavlidis P, Shumyatsky GP, Tibbs GR, Siegelbaum SA (2000) Molecular and functional heterogeneity of hyperpolarizationactivated pacemaker channels in the mouse CNS. J Neurosci 20:52645275.

Schrader LA, Ren Y, Cheng F, Bui D, Sweatt JD, Anderson AE (2009) Kv4.2 is a locus for PKC and ERK/MAPK cross-talk. Biochem J 417:705-715.

Shah MM, Migliore M, Valencia I, Cooper EC, Brown DA (2008) Functional significance of axonal Kv7 channels in hippocampal pyramidal neurons. Proc Natl Acad Sci U S A 105:7869-7874.

Spampanato J, Mody I (2007) Spike timing of lacunosom-moleculare targeting interneurons and CA3 pyramidal cells during high-frequency network oscillations in vitro. J Neurophysiol 98:96-104.

Spruston N (2008) Pyramidal neurons: dendritic structure and synaptic integration. Nat Rev Neurosci 9:206-221.

Storm JF (1988) Temporal integration by a slowly inactivating K+ current in hippocampal neurons. Nature 336:379-381.
Storm JF (1990) Potassium currents in hippocampal pyramidal cells. Prog Brain Res 83:161-187.

Surges R, Sarvari M, Steffens M, Els T (2006) Characterization of rebound depolarization in hippocampal neurons. Biochem Biophys Res Comm 348:1343-1349.

Tamás G, Lorincz A, Simon A, Szabadics J (2003) Identified sources and targets of slow inhibition in the neocortex. Science 299:1902-1905.

Taverna S, Tkatch T, Metz AE, Martina M (2005) Differential expression of TASK channels between horizontal interneurons and pyramidal cells of rat hippocampus. J Neurosci 25:9162-9170.

Ulens C, Tytgat J (2001) Functional heteromerization of HCN1 and HCN2 pacemaker channels. J Biol Chem 276:6069-6072.

van Welie I, Wadman WJ, van Hooft JA (2005) Low affinity block of native and cloned hyperpolarization-activated $\mathrm{Ih}$ channels by $\mathrm{Ba}^{2+}$ ions. Eur J Pharmacol 507:15-20.

Varga AW, Yuan LL, Anderson AE, Schrader LA, Wu GY, Gatchel JR, Johnston D, Sweatt JD (2004) Calcium-calmodulin-dependent kinase II modulates Kv4.2 channel expression and upregulates neuronal A-type potassium currents. J Neurosci 24:3643-3654.

Yuan LL, Chen X (2006) Diversity of potassium channels in neuronal dendrites. Prog Neurobiol 78:374-389.

Yuan LL, Adams JP, Swank M, Sweatt JD, Johnston D (2002) Protein kinase modulation of dendritic $\mathrm{K}^{+}$channels in hippocampus involves a mitogenactivated protein kinase pathway. J Neurosci 22:4860-4868.

Zhang W, Shin JH, Linden DJ (2004) Persistent changes in the intrinsic excitability of rat deep cerebellar nuclear neurones induced by EPSP or IPSP bursts. J Physiol 561:703-719. 